\title{
Muscle-derived stem/progenitor cell dysfunction in Zmpste24-deficient progeroid mice limits muscle regeneration
}

\author{
Minjung Song ${ }^{1 \dagger}$, Mitra Lavasani ${ }^{1,2^{*}+}$, Seth D Thompson ${ }^{1}$, Aiping Lu', Bahar Ahani ${ }^{1}$ and Johnny Huard ${ }^{1,2,3^{*}}$
}

\begin{abstract}
Introduction: Loss of adult stem cell function during aging contributes to impaired tissue regeneration. Here, we tested the aging-related decline in regeneration potential of adult stem cells residing in the skeletal muscle.

Methods: We isolated muscle-derived stem/progenitor cells (MDSPCs) from progeroid Zmpste24-deficient mice $\left(\right.$ Zmpste24 $4^{--}$) with accelerated aging phenotypes to investigate whether mutation in lamin A has an adverse effect on muscle stem/progenitor cell function.

Results: Our results indicate that MDSPCs isolated from Zmpste $24^{-/-}$mice show reduced proliferation and myogenic differentiation. In addition, Zmpste24 $4^{-1-}$ MDSPCs showed impaired muscle regeneration, with a limited engraftment potential when transplanted into dystrophic muscle, compared with wild-type (WT) MDSPCs. Exposure of progeroid Zmpste24 $4^{-1-}$ MDSPCs to WT MDSPCs rescued the myogenic differentiation defect in vitro.

Conclusions: These results demonstrate that adult stem/progenitor cell dysfunction contributes to impairment of tissue regeneration and suggest that factors secreted by functional cells are indeed important for the therapeutic effect of adult stem cells.
\end{abstract}

\section{Introduction}

Hutchinson-Gilford progeria syndrome (HGPS) is an autosomal dominant disease that involves premature aging, causing early death in childhood due to stroke or myocardial infarction. The patients have sclerotic skin, joint contractures, bone abnormalities, and growth impairment [1]. A point mutation of the lamin A gene (LMNA), which encodes lamin A protein, was found to be the main cause of HGPS [2,3]. Lamin A is a nuclear envelope protein that gives structural support to the nucleus and is involved in various cellular roles, such as gene expression and DNA replication $[4,5]$.

Murine models of HGPS have been created by altering posttranslational modification steps of lamin A [6-8]. Young and colleagues [6] developed Zmpste24 knockout mice with many features common to HGPS [6].

\footnotetext{
*Correspondence: mil39@pitt.edu; jhuard@pitt.edu

+ Contributed equally

'Stem Cell Research Center, Department of Orthopaedic Surgery, University of Pittsburgh School of Medicine, 450 Technology Drive, Suite 206, Pittsburgh, PA 15219, USA

Full list of author information is available at the end of the article
}

ZMPSTE24 is a metalloproteinase required for cleaving the carboxylic group of prelamin A to create lamin A. By knocking out Zmpste24, prelamin A accumulates on the cell nuclear envelope, resulting in cellular blebbing $[9,10]$. The Zmpste $24^{-1-}$ mice display accelerated aging, loss of weight, spontaneous bone fracture, cardiomyopathy, muscular dystrophy, muscle atrophy, and muscle weakness $[6,7,9,11]$. A recent study provides evidence that the skeletal muscles of Zmpste24 ${ }^{-1-}$ mice exhibit impaired muscle contraction and neuromuscular performance [11].

Prelamin A has been shown to be involved in early steps of $\mathrm{C} 2 \mathrm{C} 12$ myoblasts differentiation [12]. C2C12 myoblasts expressing mutated lamin A have also demonstrated a reduced capacity to undergo myogenic differentiation [13]. Downregulation of lamin A/C levels in myoblasts, by transfecting them with a mutant Lmna gene, isolated from Lmna-knockout mice, or from silencing RNA targeting A-type lamins, showed impaired differentiation kinetics and reduced differentiation potential $[14,15]$. These data provide evidence for a critical role of prelamin A in the early steps of muscle cell differentiation.
Ciomed Central

() 2013 Song et al.; licensee BioMed Central Ltd. This is an open access article distributed under the terms of the Creative Commons Attribution License (http://creativecommons.org/licenses/by/2.0), which permits unrestricted use, distribution, and reproduction in any medium, provided the original work is properly cited. 
MDSPCs are an important population of adult stem cells isolated from skeletal muscle by using a modified preplate technique $[16,17]$. They show self-renewable potential and multilineage differentiation for myogenic, osteogenic, chondrogenic, and adipogenic lineages in vitro [16,18-21]. Prominent muscle regeneration has been observed with MDSPCs transplantation into a dystrophin knockout mouse model of Duchenne muscular dystrophy $(m d x)[16,22,23]$. Although adult stem cells play essential roles in maintaining tissue and organ function with selfrenewal and multilineage differentiation potential, they also show age-dependent changes, such as decline in number and function similar to other somatic cells [24]. Given that stem cell exhaustion and loss of function with age may limit their muscle-regeneration potential, we investigated the impact of aging on MDSPC function by using progeroid Zmpste24-deficient mice, which exhibit accelerated aging and mimic HGPS.

\section{Methods}

Zmpste24-- mice

Zmpste $24^{-/-}$genotyping was performed with polymerase chain reaction (PCR) with oligonucleotides forward: 5'-TCACATGGAGTGAATGCTCTG-3' and reverse: 5'-AGTGAACACCAGGCCAGTTT-3' [6]. All animal experiments were performed in accordance with the Institutional Animal Care and Use Committee of the University of Pittsburgh.

\section{MDSPC isolation}

MDSPCs were isolated from 8-week-old Zmpste24 $4^{-1-}$ mice and WT littermates by using a modified preplate technique according to a previously established protocol $[16,17]$. MDSPCs were cultured in proliferation medium (PM) containing Dulbecco's modified Eagles medium (DMEM, high glucose) supplemented with 10\% horse serum, 10\% fetal bovine serum (FBS), 1\% penicillin-streptomycin (all from Invitrogen, Grand Island, NY, USA), and 0.5\% chick embryo extract (Accurate Chemical, Westbury, NY, USA), on collagen type I-coated flasks (Sigma-Aldrich, St. Louis, MO, USA). Cells were used between passages 20 and 30.

\section{Proliferation in vitro}

The population doubling time (PDT) over a 72-hour period was measured from time-lapse images acquired through a live-cell imaging (LCI) system (Automated Cell Technologies, Inc., Pittsburgh, PA, USA), as previously described $[25,26]$. In brief, cells were plated at an initial density of 2,000 cells/well in collagen type I-coated 24-well plates, and images were acquired at 15-minute intervals over a period of 3 days by using an LCI system equipped with a $10 \times$ objective. Five images at each time point were randomly selected and analyzed, by using Image J software (v. 1.44p; National Institutes of Health,
Bethesda, MD, USA), from three independent experiments on three distinct cell lines in each group.

\section{Myogenic differentiation in vitro}

MDSPCs were seeded onto collagen type I-coated six-well plates $\left(1 \times 10^{5}\right.$ cells/well $)$ and cultured for 2 days in PM. Myogenic differentiation was induced by replacing the PM with differentiation media (DMEM, 2\% FBS, $1 \%$ penicillinstreptomycin) and cultured for 3 days. Immunostaining for fast myosin heavy chain (f-MyHC), a marker of terminal myogenic differentiation, was performed to stain differentiated muscle cells. Cells were fixed with $-20^{\circ} \mathrm{C}$ methanol, blocked with $5 \%$ goat serum, and incubated at room temperature with primary mouse anti-MyHC fast (1:250; Sigma-Aldrich) and secondary biotinylated IgG (1:250; Vector Laboratories, Burlingame, CA, USA) each for 1 hour, and streptavidin-594 (1:500; Sigma-Aldrich) for 15 minutes. The nuclei were visualized by staining with DAPI (4', 6' diamidino-2-phenylindole, 100 ng/ml; SigmaAldrich) for 10 minutes. Fluorescent images were taken by using a Leica DMIRB inverted microscope equipped with a QImaging Retiga digital camera with Northern Eclipse software (v. 6.0; Empix Imaging, Inc., Cheektowaga, NY, USA). Total cell nuclei and nuclei within f-MyHC-positive myofibers, from at least 15 fields, each from three replica platings, were counted by using Image). The myogenic differentiation was calculated as percentage of cells expressing f-MyHC (red) per total nuclei (DAPI, blue).

\section{Western blot}

MDSPCs from WT and Zmpste24//- were lysed in RadioImmunoprecipitation Assay (RIPA) buffer (SigmaAldrich). Protein concentration was measured with the Bradford protein assay reagent (Bio-Rad, Hercules, CA, USA). Equal amounts of protein from each sample were loaded on $10 \%$ SDS-polyacrylamide gels and run for 2 hours at 100 volts. Proteins were then transferred for 60 minutes by 100 volts to a nitrocellulose membrane (Millipore, Billerica, MA, USA) and blocked by $5 \%$ nonfat dry milk (Bio-rad) in Tris-buffered saline Tween-20 (TBST) for 1 hour. Samples were probed with goat Nterminal anti-lamin A/C (1:200, sc-6215), goat C-terminal anti-prelamin A (1:200, sc-6214), and rabbit anti- $\beta$-actin (1:200, all from Santa Cruz Biotechnology, Inc., Dallas, TX, USA), overnight at $4^{\circ} \mathrm{C}$. After washing 3 times with TBST, the membrane was incubated with secondary antibody rabbit anti-goat IgG (H\&L) coupled to horseradish peroxidase (HRP; Santa Cruz Biotechnology, Inc.) and mouse anti-rabbit IgG-HRP for 1 hour at room temperature. Blots were developed by using a SuperSignal West Femto with enhanced chemiluminescent substrate (1:100,000; Thermo Scientific, Rockford, IL, USA), and the bands were detected on $\mathrm{x}$-ray film. 
Reverse transcriptase polymerase chain reaction (RT-PCR) RNA was extracted from cultured cells by using TRIzol (Invitrogen) and a RNeasy Mini kit (Qiagen Inc., Hilden, Germany). The amount of RNA yield was quantified by using a Nano-Quant (Tecan, San Jose, CA, USA), and a total of $1 \mu \mathrm{g}$ of RNA was reverse-transcribed into cDNA by using the Superscript III Reverse Transcriptase (Invitrogen), according to the manufacturer's instructions. Then, cDNA was mixed with GoTaq polymerase, dNTPs, and green GoTag reaction buffer (Promega, Madison, WI, USA) and amplified on a thermal cycler (Eppendorf Mastercycler proS), according to the manufacturer's instructions for 30 cycles at $58^{\circ} \mathrm{C}$ annealing temperature. PCR products were separated by using $2 \%$ agarose gels with $1 \%$ ethidium bromide. The gel images were captured with a BioRad Gel Doc system. The following primers were used: Stem cell antigen-1 (Sca-1), forward 5'-CCTACTGTGTGCAGAAAGAGC-3' and reverse 5'-CAGGAAGTCTTCACGTTGACC-3'; CD34, forward 5'-GCAGCTTTGAG ATGACATCACC-3' and reverse 5'-CTCAGCCTCCTC CTTTTCACA-3'; myogenin, forward 5'-CTACAGGCCT TGCTCAGCTC-3' and 5'-TTGTGGGCGTCTGTAGG3'; desmin, forward 5'-AACCTGATAGACGACCTGC AG-3' and reverse 5'-GCTTGGACATGTCCATCTCCA3'. $\beta$-actin, forward 5'-GGGTCAGAAGGACTCCTATG-3' and reverse 5'-CTTTGATGTCACGCAGCACGATT-3' was used as a loading control.

\section{Coculture experiments}

Zmpste24 $4^{-1-}$ MDSPCs were plated in the lower compartment of Costar Transwell Permeable Supports (Corning, Tewksbury, MA, USA) in PM at a density of 3,000 cells/ well in a 24-well collagen type I-coated plate. WT MDSPCs were seeded into 6.5- $\mathrm{mm}$ transwell membrane inserts at the same density in PM and placed above the Zmpste $24^{-1-}$ MDSPCs. The 24-well plates containing the transwells were then placed in the LCI system for 72 hours to measure proliferation of the $\mathrm{Zmpste} \mathrm{4}^{-1-}$ MDSPCs, as described earlier. As a control, each plate contained wells of Zmpste $24^{-1-}$ MDSPCs with empty transwell membrane inserts. To measure the differentiation potential of Zmpste $24^{-1-}$ MDSPCs after coculture, transwell inserts were removed after 72 hours, and the PM media was switched to differentiation media. After 2 to 3 days, myogenic differentiation of the cells was tested by immunostaining for $\mathrm{f}-\mathrm{MyHC}$, as described earlier. Changes in differentiation potential of Zmpste $24^{-/-}$MDSPCs was also tested by using conditioned media from the WT MDSPCs. WT MDSPCs were cultured for 2 days in $25 \mathrm{~cm}^{2}$ collagen-coated flasks and were then treated with differentiation media for 3 days. The supernatant was collected and used as conditioned media for 8-week-old Zmpste $24^{-1-}$ MDSPCs, whereas unconditioned differentiation media was used as a control.

\section{Cell transplantation}

The $3 \times 10^{5}$ donor MDSPCs isolated from WT or Zmpste $24^{-1-}$ mice were intramuscularly transplanted into the gastrocnemius muscles of 8 - to 9 -week-old $m d x /$ SCID mice (C57BL/10ScSn DMDmdx/J/CB17-Prkdcscid/J; The Jackson Laboratory), an animal model of Duchenne muscular dystrophy that is also immunocompromised. Mice were killed 2 weeks after transplantation, and the gastrocnemius muscles $(n=3)$ were harvested, frozen in 2-methylbutane pre-cooled in liquid nitrogen, and cryosectioned $(10 \mu \mathrm{m})$. Sections were immunostained for dystrophin, the cross-sectional area of dystrophin-positive myofibers was measured, and the distribution of the fiber areas was plotted as previously described $[27,28]$.

\section{Statistical analysis}

Statistical analyses were carried out by using Sigmastat (Jandel Scientific, v. 2.0, San Rafael, CA, USA) software package. The Student's $t$ test or Mann-Whitney Rank Sum test was used for direct comparisons between groups. For multiple comparisons, the one-way ANOVA or the Kruskal-Wallis one-way ANOVA on ranks was applied. Pairwise multiple comparisons were performed by using the Tukey's Test after rank-based ANOVA. All values are expressed as the mean $\pm \mathrm{SD}$, and $P<0.05$ was considered significant.

\section{Results}

MDSPCs from accelerated aged mice show defect in prelamin A processing and stem cell marker expression

To determine whether a defect in lamin A/C processing, previously reported in Zmpste24-deficient mice [7,10], affects our stem/progenitor cells, MDSPCs isolated from 8-week-old WT and Zmpste24-deficient mice were evaluated for expression and distribution of lamin $\mathrm{A} / \mathrm{C}$ and prelamin A (Figure 1A). An antibody specific to prelamin $\mathrm{A}$ and lamin $\mathrm{A} / \mathrm{C}$ revealed accumulation of prelamin Aand an absence of lamin A expression in the Zmpste $24^{-1-}$ MDSPCs in comparison with WT MDSPCs. These results confirm the lack of prelamin A processing in Zmpste24deficient MDSPCs, as previously reported in other cells isolated from these mutant mice [9,29]. Stem cell potential was also investigated by measuring the expression levels of CD34 and Sca-1 for each cell population [27]. Zmpste24deficient MDSPCs show relatively lower expression levels of CD34 compared with WT MDSPCs; however, the expression levels of Sca-1 were similar in both groups (Figure 1B).

\section{Zmpste24/- MDSPCs show slower proliferation}

Proliferation kinetics of MDSPCs isolated from WT and Zmpste24-deficient mice was measured in vitro by using an LCI system, and their numbers per image were manually counted. Analysis of images showed that the proliferation 


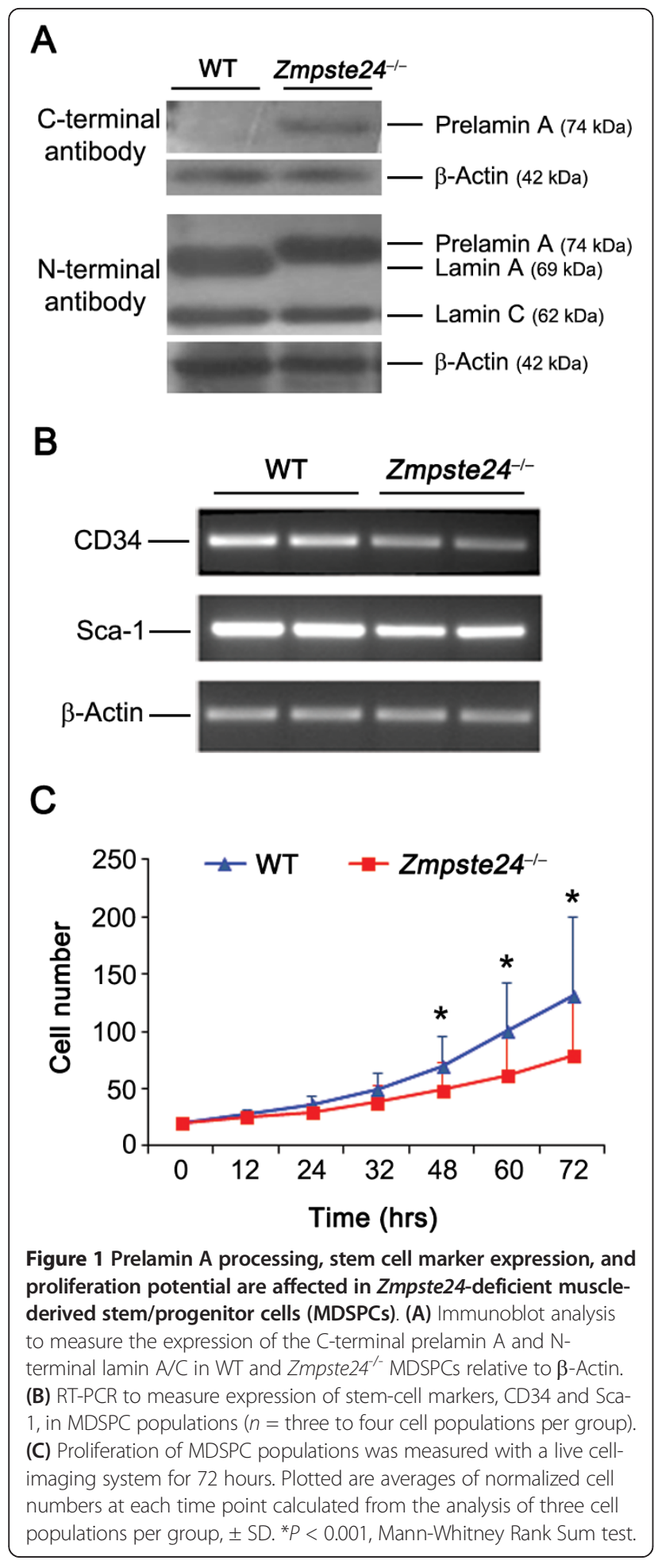

rate of WT and Zmpste24 ${ }^{-1-}$ MDSPCs were similar for the first 24 hours (Figure 1C). However, Zmpste24 $4^{-/-}$MDSPCs started to show a trend toward a reduction in proliferation rate after 48 hours, which became significant at 72 hours compared with WT MDSPCs ( ${ }^{*} P<0.05$, Figure $\left.1 C\right)$. These data demonstrate that the proliferation capacity of MDSPCs diminishes in Zmpste24-deficient mice, as observed in aged MDSPCs [30].

\section{Zmpste24 $4^{-/-}$MDSPCs display limited myogenic differentiation capacity}

To determine whether the myogenic differentiation capacity of Zmpste $24^{-1-}$ MDSPCs is affected, the expression levels of essential proteins that play critical roles in regulating at least one component of myogenic terminal differentiation were investigated. Myogenin is a muscle-specific transcription factor involved in functional skeletal muscle development and is expressed mainly in intermediate stages of myogenesis [31]. Desmin is expressed at early stages of myogenic differentiation and continuously expressed near to terminal myogenesis [32]. Interestingly, the expression levels of myogenin and desmin were similar in Zmpste 24 $4^{-1-}$ MDSPCs compared with control WT MDSPCs, indicating that MDSPCs from aged mice have similar myogenic potential at the transcriptional level (Figure 2A). To test whether impairment may occur during the posttranslational step of myogenesis, cells were cultured to confluence and switched to differentiation media, and immunostained for the terminal myogenic differentiation marker, f-MyHC. The WT MDSPCs fused to form elongated multinucleated myotubes expressing $\mathrm{f}-\mathrm{MyHC}$, whereas Zmpste $24^{-/-}$MDSPCs formed significantly fewer and smaller myotubes, indicating impaired differentiation $(* P<0.001$, Figure 2B, C). Collectively, these data establish that the function of the MDSPCs is compromised in Zmpste24 $4^{-1-}$ mice, as observed in old WT MDSPCs [30].

\section{Zmpste24 ${ }^{-1-}$ MDSPCs show limited muscle regeneration}

To investigate the myogenic potential of progeroid Zmpste24/- MDSPCs in vivo, $3 \times 10^{5}$ viable MDSPCs isolated from WT and Zmpste $24^{-/-}$mice were injected into the gastrocnemius muscle of 8-week-old $m d x /$ SCID mice, a mouse model of Duchenne muscular dystrophy that lacks dystrophin at the sarcolemma of muscle fibers [33] and is also immunoincompetent. The average number of regenerated dystrophin-positive myofibers did not significantly differ between the WT $(625, n=3)$ and Zmpste24 ${ }^{-1-}(508$, $n=3)$ MDSPCs $(P=0.80)$. However, a dramatic difference was noted in the average cross-sectional area of the regenerated myofibers in mice injected with Zmpste24-deficient MDSPCs compared with WT MDSPC-injected mice (Figure 3A). Most of the regenerated myofibers from the WT MDSPC transplantations had an area of $>500 \mu \mathrm{m}^{2}$, indicative of significantly more mature regenerated myofibers. Conversely, dystrophin-positive myofibers in mice injected with Zmpste24-/- MDSPCs predominantly had an area of $\leq 500 \mu \mathrm{m}^{2}\left({ }^{*} P<0.001\right.$, Figure 3B). Dystrophinpositive centronucleated myofibers of small size regenerated by Zmpste $24^{-1-}$ MDSPC transplantation are perhaps 


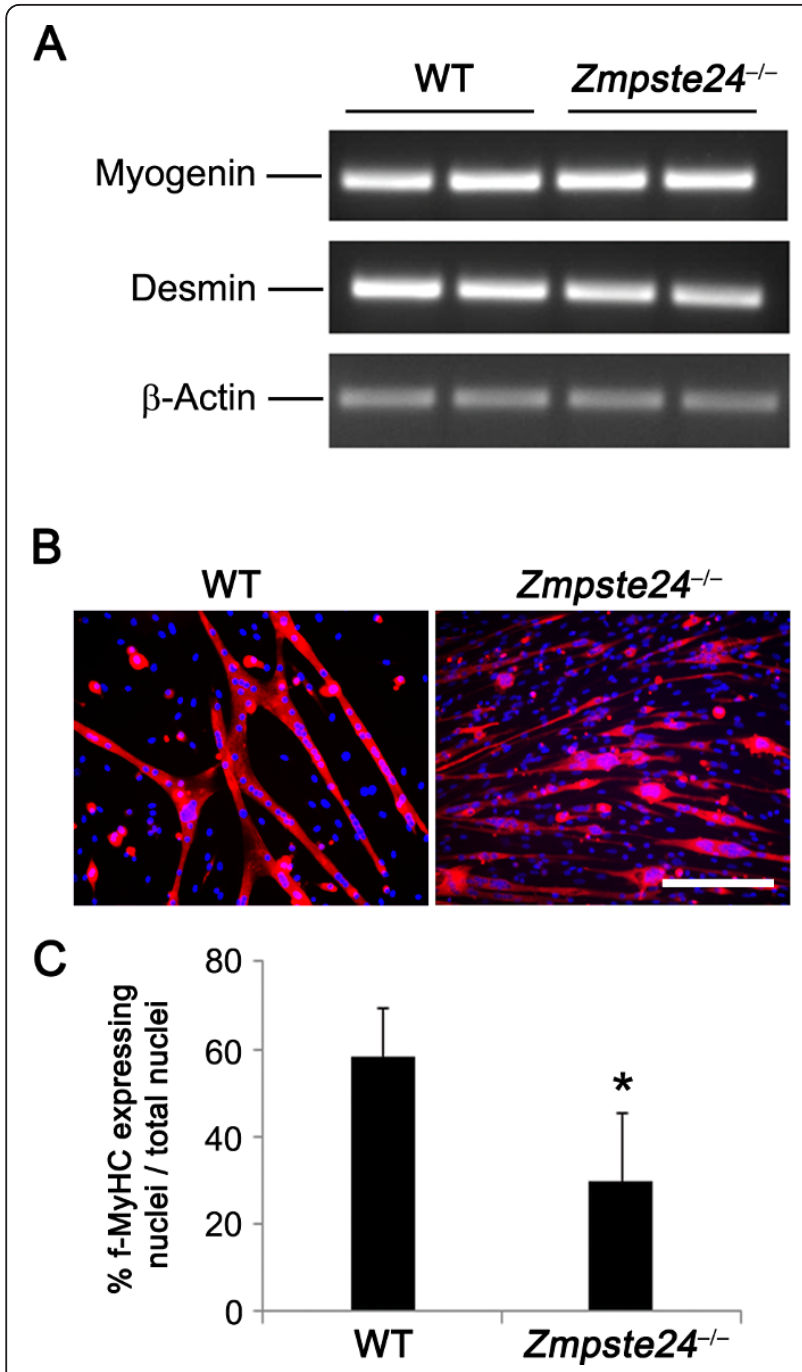

Figure 2 Myogenic differentiation potential is reduced in Zmpste24 ${ }^{-/-}$muscle-derived stem/progenitor cells (MDSPCs). (A) RT-PCR to measure the expression of the myogenic differentiation markers, myogenin and desmin. (B) Representative images of in vitro MDSPC myogenic differentiation. MDSPC populations were grown to confluence and then switched to differentiation media. After 2 to 3 days, cells were immunostained for the terminal differentiation myogenic marker, f-MyHC (red). Scale bar, $100 \mu \mathrm{m}$. (C) Graph shows the myogenic differentiation (percentage of cells (DAPI, blue) expressing f-MyHC (red)) of MDSPCs isolated from Zmpste24 $4^{-1-}$-deficient mice relative to young WT MDSPCs. Error bars indicate SD. ${ }^{*} P<0.001$, Student's $t$ test. Representative images are shown from three separate experiments using three independent MDSPC populations of each group.

due to fusion of donor cells with one another or with smaller host-regenerated myofibers. Collectively, the data in Figures 2 and 3 support the conclusion that the loss of stem cell function in the Zmpste24-deficient model of accelerated aging limits muscle regeneration.
WT MDSPCs rescue the myogenic differentiation defect of Zmpste24 ${ }^{-/}$MDSPCs

Next, we tested whether WT MDSPCs can restore the myogenic dysfunction of Zmpste24/- MDSPCs. MDSPCs isolated from Zmpste24-deficient mice were cocultured with the WT MDSPCs by using a transwell system, or in the presence of conditioned media from WT MDSPCs, and were switched to low-serum media to evaluate the myogenic differentiation. Zmpste24 $4^{-1-}$ MDSPCs tend to fuse and form spheres (f-MyHC-positive) containing single or many nuclei (DAPI) (Figure 4A). In contrast, by using the conditioned media from WT MDSPCs or after coculturing the Zmpste24 $4^{-/-}$MDSPCs with WT MDSPCs, normal myotube formation was restored, indicated by numerous elongated multinucleated myotubes generated by the rescued Zmpste24-1- MDSPCs (Figure 4A). The differentiation defect was reported by using the observation that Zmpste24 ${ }^{-/-}$MDSPCs formed more mononucleated and immature myotubes (myotubes containing two to three nuclei and expressing $\mathrm{f}-\mathrm{MyHC}$ ) compared with WT MDSPCs forming more mature myotubes (containing four or more nuclei; ${ }^{*} P<0.05$ ). Analyzing the type of $\mathrm{f}-\mathrm{MyHC}$ expressing myotubes revealed a significant shift in the distribution to more mature myotube formation when Zmpste24 $4^{-1-}$ MDSPCs were grown in the presence of the conditioned media isolated from WT MDSPC cultures $\left({ }^{\#} P<0.05\right.$, relative to WT MDSPCs) or cocultured with transwells containing the WT MDSPCs $\left({ }^{\mathbb{P}} P<0.05\right)$ relative to Zmpste24 $4^{-1-}$ MDSPCs alone or WT MDSPCs (Figure 4B). Thus WT MDSPCs were able to rescue, at least in part, the myogenic differentiation capacity of MDSPCs isolated from $Z$ mpste $24^{-1-}$ progeroid mice.

\section{Discussion}

Numerous studies have reported that mutation of lamin A, a major component of the nuclear envelope, has an adverse effect on skeletal muscle function and myoblast differentiation $[7,9,11-14,34]$; however, no studies examined earlier muscle stem/progenitor cells residing in muscle tissue. Because degenerative changes associated with progeroid syndromes may have a strong impact on stem cells, we hypothesized that the dysfunction of muscle stems cells in Zmpste $24^{-1-}$ progeroid mice may contribute to the loss of the cells' ability to regenerate skeletal muscle. To evaluate this hypothesis, we isolated MDSPCs from Zmpste $24^{-1-}$ mice showing nuclear lamina defects with accelerated aging phenotypes. Our results show that expression of stem cell markers decreased in MDSPCs isolated from skeletal muscle of Zmpste $24^{-/-}$progeroid mice in comparison with their WT littermates. Furthermore, MDSPC proliferation and myogenic differentiation was significantly impaired in Zmpste24/- MDSPCs relative to 


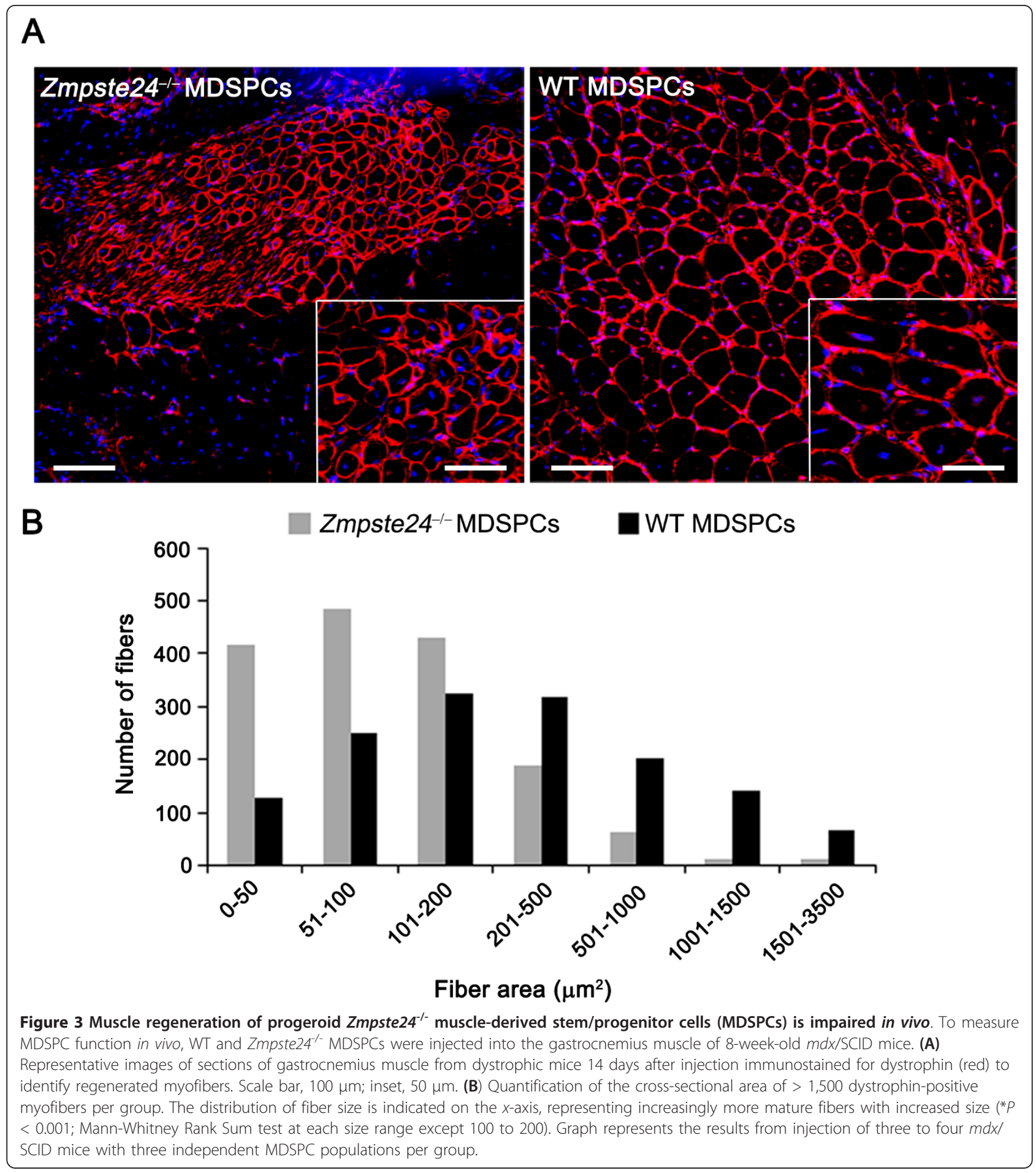

MDSPCs isolated from WT mice. This is consistent with our recent findings using naturally aged and excision repair cross-complementation group 1 (ERCC1)-deficient mice, a mouse model of progeroid syndrome, indicating that MDSPCs isolated from aged and progeroid mice showed loss-of-stemness properties including proliferation and multilineage differentiation [30]. A recent report also showed an alteration in the number and proliferation capacity of epidermal stem cells in $Z$ mpste $24^{-1-}$ progeroid mice [29]. Similar parallels were found in the vascular smooth muscles and mesenchymal stem cells (MSCs) compartment of induced pluripotent stem cells (iPSCs) 


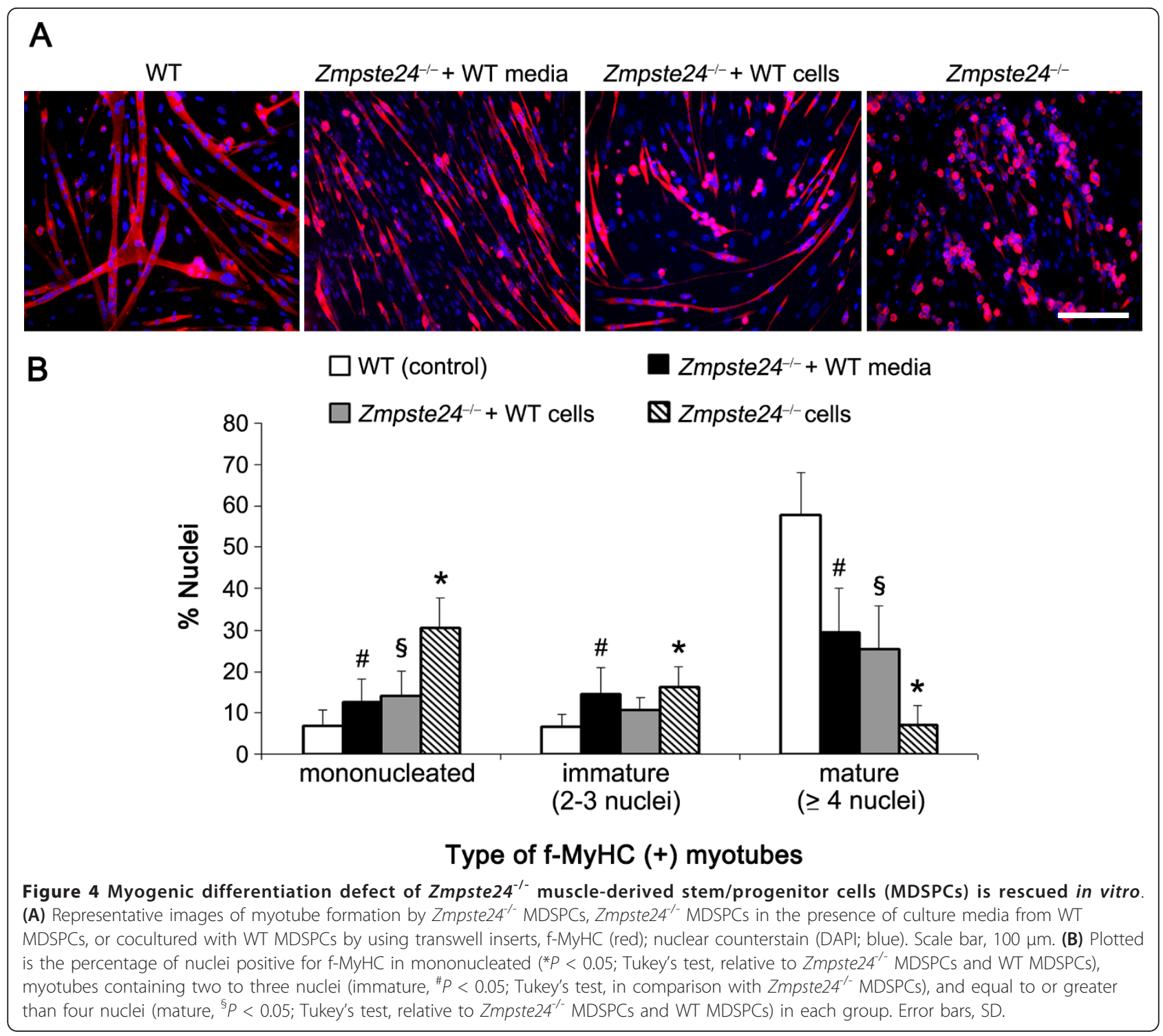

from HGPS dermal fibroblasts [35]. Similarly, molecular and cellular identity, including the differentiation potential, were altered in human MSCs (hMSCs) isolated from HGPS patients [36].

Interestingly, the expression levels of myogenic markers, myogenin, and desmin, were not affected in MDSPCs isolated from Zmpste $24^{-1-}$ mice, whereas the terminal myogenic differentiation marker, $\mathrm{f}-\mathrm{MyHC}$, was significantly impaired. This suggests that the dysfunction of MDSPCs is most likely due to changes in the posttranscriptional stages of myogenic differentiation. Recent studies suggested important findings regarding different signaling pathways involved in the regulation of stem-cell function. Signaling pathways essential for the maintenance of stem cell fate and function, such as Wnt in epidermal cells and microphthalmia transcription factor (Mitf) in melanocytes, has been shown to be defective in Zmpste24 $4^{-/-}$mice [29]. Lineage-specific differentiation of hMSCs was altered through the activation of the Notch signaling pathway, a pathway known to regulate stem-cell differentiation [36]. Therefore, future studies are necessary to elucidate the role of lamin A and possible changes in the signaling pathways of MDSPCs isolated from Zmpste24/- progeroid mice.

Our transplantation study showed that the muscleregeneration potential of Zmpste24-1- MDSPCs was significantly reduced in comparison to MDSPCs isolated from WT mice. This supports our hypothesis that loss of stem-cell function diminishes their regenerative potential. These results also agree with our recent findings that muscle from aged and progeroid ERCC1-deficient mice had significantly reduced regenerative capacity after 
cardiotoxin-induced injury [30]. This is consistent with the notion that defects in the adult stem-cell compartment, which accrue over time, contribute to the loss of tissue-regeneration capacity and homeostasis associated with aging. In addition to the cell-intrinsic defect, local microenvironment/niche and systemic environment have also been recognized to play a role in stem-cell aging [24]. Muscle stem cells interact with a microenvironment comprising of myofibers and the basal lamina. Therefore, the microenvironment of the $m d x /$ SCID mouse skeletal muscle most likely could not support the effective regeneration of the progeroid MDSPCs, reinforcing the important role of a healthy microenvironment to promote successful tissue regeneration.

Notably, the ability of Zmpste24-/- MDSPCs to undergo myogenic differentiation was rescued after coculture and in the presence of the WT MDSPC conditioned media. This result is similar to recent reports showing that WT MDSPCs rescue differentiation defects of MDSPCs isolated from naturally aged and progeroid ERCC1-deficient mice when cocultured [30]. These results demonstrate that dysfunction of progeroid stem cells can be rescued, at least in part, by exogenous factors secreted by the WT stem cells. Although the exact secreted factors involved have yet to be elucidated, several candidate proteins and signaling pathways are currently under investigation. Similar results were found in vivo by Conboy and colleagues [37] by using parabiosis, in which functional defects and the regenerative capacity of satellite cells in aged mice were restored by secreted factors from a young systemic environment. Together, these findings suggest the possibility of epigenetic changes of stem and progenitor cells in response to environmental cues. It will be of great interest to identify the factors secreted by tissuespecific host cells in response to transplanted donor cells.

Finally, limitation of progeroid MDSPCs to undergo myogenic differentiation, at least in part, may explain the muscle atrophy and weakness observed in Zmpste24 $4^{-/-}$ progeroid mice. In addition, the reversal of the phenotypic defects of stem cells with coculture can provide future opportunities for therapeutic strategies.

\section{Conclusions}

The present study demonstrates that MDSPCs isolated from Zmpste $24^{-1-}$ progeroid mice are defective in their proliferation and differentiation capabilities in culture and during tissue regeneration. It remains unclear whether the defects in MDSPC function arise because of defects in the stem cells and/or the stem-cell niche. Therefore, future studies are needed to investigate the mechanism(s) that confer MDSPC dysfunction with aging. In addition, myogenic differentiation of defective MDSPCs was improved after coculturing the cells with young functional WT MDSPCs. Hence, identifying the factors secreted by the functional stem cells responsible for rejuvenation will be valuable for overcoming muscular degeneration commonly associated with muscular dystrophies and aging.

\section{Abbreviations}

ANOVA: analysis of variance; CDNA: complementary deoxyribonucleic acid; DAPI: 4',6' diamidino-2-phenylindole; DMEM: Dulbecco's modified Eagle medium; dNTP: deoxyribonucleotide triphosphate; ERCC1: excision repair cross-complementation group 1; FBS: fetal bovine serum; f-MyHC: fast myosin heavy chain; HGPS: Hutchinson-Gilford progeria syndrome; hMSC: human mesenchymal stem cell; HRP: horseradish peroxidase; iPSC: induced pluripotent stem cell; LCl: live cell imaging; LMNA: lamin A gene; MDSPC: muscle-derived stem/progenitor cell; Mitf: microphthalmia transcription factor; PCR: polymerase chain reaction; PDT: population doubling time; PM: proliferation media; RIPA: radio-immunoprecipitation assay; RNA: ribonucleic acid; RT-PCR: reverse transcriptase polymerase chain reaction; Sca-1: stem cell antigen-1; SCID: severe combined immune deficiency; SDS: sodium dodecylsulfate; TBST: tris-buffered saline tween 20; WT: wild type.

\section{Authors' contributions}

MS designed experiments, carried out proliferation and myogenic differentiation assays, analyzed data, and drafted the manuscript. ML carried out coculture experiments and in vivo experiments, analyzed data, performed the statistical analyses, and wrote the manuscript. ST assisted with in vivo studies, image analysis, and revised the manuscript. AL isolated the stem-cell lines and provided assistance with experimental design. BA carried out all of the immunostaining, imaging, and quantification. $\mathrm{JH}$ supervised the design and execution of the experiments. All the authors read and approved the manuscript for publication.

\section{Competing interests}

Johnny Huard receives consulting fees and royalties from Cook MyoSite Inc. All the other authors declare that they have no competing interests.

\section{Acknowledgements}

We thank Jonathan D. Proto and Ying Tang for the helpful discussion regarding the RT-PCR and Jim Cummins for editing. Zmpste $24^{-/-}$mice were obtained from Dr. Young at the University of California, Los Angeles [10], and muscle tissues were provided by Dr. Orlic at the NIH. This work was supported in part by NIH 1R21AG033907-01A1, the Henry J. Mankin Endowed Chair at the University of Pittsburgh, and the William F. and Jean W. Donaldson endowed chair at the Children's Hospital of Pittsburgh.

\section{Author details}

${ }^{1}$ Stem Cell Research Center, Department of Orthopaedic Surgery, University of Pittsburgh School of Medicine, 450 Technology Drive, Suite 206, Pittsburgh, PA 15219, USA. ²Department of Bioengineering University of Pittsburgh Swanson School of Engineering, 300 Technology Drive, Suite 360B, Pittsburgh, PA 15219, USA. ${ }^{3}$ Department of Microbiology and Molecular Genetics, University of Pittsburgh School of Medicine, 450 Technology Drive, Suite 523, Pittsburgh, PA 15219, USA.

Received: 31 May 2012 Revised: 18 January 2013 Accepted: 13 February 2013 Published: 25 March 2013

\section{References}

1. Merideth MA, Gordon LB, Clauss S, Sachdev V, Smith AC, Perry MB, Brewer CC, Zalewski C, Kim HJ, Solomon B, Brooks BP, Gerber LH, Turner ML, Domingo DL, Hart TC, Graf J, Reynolds JC, Gropman A, Yanovski JA, Gerhard-Herman M, Collins FS, Nabel EG, Cannon RO, Gahl WA, Introne WJ: Phenotype and course of Hutchinson-Gilford progeria syndrome. N Engl J Med 2008, 358:592-604.

2. Eriksson M, Brown WT, Gordon LB, Glynn MW, Singer J, Scott L, Erdos MR, Robbins CM, Moses TY, Berglund P, Dutra A, Pak E, Durkin S, Csoka AB, Boehnke M, Glover TW, Collins FS: Recurrent de novo point mutations in lamin A cause Hutchinson-Gilford progeria syndrome. Nature 2003, 423:293-298. 
3. De Sandre-Giovannoli A, Bernard R, Cau P, Navarro C, Amiel J, Boccaccio I, Lyonnet S, Stewart CL, Munnich A, Le Merrer M, Levy N: Lamin a truncation in Hutchinson-Gilford progeria. Science 2003, 300:2055.

4. Sullivan T, Escalante-Alcalde D, Bhatt H, Anver M, Bhat N, Nagashima K, Stewart CL, Burke B: Loss of A-type lamin expression compromises nuclear envelope integrity leading to muscular dystrophy. J Cell Biol 1999, 147:913-920.

5. Goldman RD, Shumaker DK, Erdos MR, Eriksson M, Goldman AE, Gordon LB, Gruenbaum Y, Khuon S, Mendez M, Varga R, Collins FS: Accumulation of mutant lamin A causes progressive changes in nuclear architecture in Hutchinson-Gilford progeria syndrome. Proc Natl Acad Sci USA 2004, 101:8963-8968.

6. Leung GK, Schmidt WK, Bergo MO, Gavino B, Wong DH, Tam A, Ashby MN, Michaelis S, Young SG: Biochemical studies of Zmpste24-deficient mice. J Biol Chem 2001, 276:29051-29058.

7. Bergo MO, Gavino B, Ross J, Schmidt WK, Hong C, Kendall LV, Mohr A, Meta M, Genant H, Jiang Y, Wisner ER, Van Bruggen N, Carano RA, Michaelis S, Griffey SM, Young SG: Zmpste24 deficiency in mice causes spontaneous bone fractures, muscle weakness, and a prelamin A processing defect. Proc Natl Acad Sci USA 2002, 99:13049-13054.

8. Mounkes LC, Kozlov S, Hernandez L, Sullivan T, Stewart CL: A progeroid syndrome in mice is caused by defects in A-type lamins. Nature 2003, 423:298-301.

9. Pendas AM, Zhou Z, Cadinanos J, Freije JM, Wang J, Hultenby K, Astudillo A Wernerson A, Rodriguez F, Tryggvason K, Lopez-Otin C: Defective prelamin A processing and muscular and adipocyte alterations in Zmpste24 metalloproteinase-deficient mice. Nat Genet 2002, 31:94-99.

10. Fong LG, Ng JK, Meta M, Cote N, Yang SH, Stewart CL, Sullivan T, Burghardt A, Majumdar S, Reue K, Bergo MO, Young SG: Heterozygosity for Lmna deficiency eliminates the progeria-like phenotypes in Zmpste24deficient mice. Proc Natl Acad Sci USA 2004, 101:18111-18116.

11. Greising SM, Call JA, Lund TC, Blazar BR, Tolar J, Lowe DA: Skeletal muscle contractile function and neuromuscular performance in Zmpste24 (-/-) mice, a murine model of human progeria. Age (Dordr) 2012, 34:805-819.

12. Capanni C, Del Coco R, Squarzoni S, Columbaro M, Mattioli E, Camozzi D, Rocchi A, Scotlandi K, Maraldi N, Foisner R, Lattanzi G: Prelamin A is involved in early steps of muscle differentiation. Exp Cell Res 2008, 314:3628-3637.

13. Favreau C, Higuet D, Courvalin JC, Buendia B: Expression of a mutant lamin A that causes Emery-Dreifuss muscular dystrophy inhibits in vitro differentiation of C2C12 myoblasts. Mol Cell Biol 2004, 24:1481-1492.

14. Frock RL, Kudlow BA, Evans AM, Jameson SA, Hauschka SD, Kennedy BK: Lamin $A / C$ and emerin are critical for skeletal muscle satellite cell differentiation. Genes Dev 2006, 20:486-500.

15. Markiewicz $E$, Ledran $M$, Hutchison CJ: Remodelling of the nuclear lamina and nucleoskeleton is required for skeletal muscle differentiation in vitro. J Cell Sci 2005, 118:409-420.

16. Qu-Petersen Z, Deasy B, Jankowski R, Ikezawa M, Cummins J, Pruchnic R, Mytinger J, Cao B, Gates C, Wernig A, Huard J: Identification of a novel population of muscle stem cells in mice: potential for muscle regeneration. J Cell Biol 2002, 157:851-864.

17. Gharaibeh B, Lu A, Tebbets J, Zheng B, Feduska J, Crisan M, Peault B, Cummins J, Huard J: Isolation of a slowly adhering cell fraction containing stem cells from murine skeletal muscle by the preplate technique. Nat Protocol 2008, 3:1501-1509.

18. Peng H, Wright V, Usas A, Gearhart B, Shen HC, Cummins J, Huard J: Synergistic enhancement of bone formation and healing by stem cellexpressed VEGF and bone morphogenetic protein-4. J Clin Invest 2002, 110:751-759.

19. Deasy BM, Gharaibeh BM, Pollett JB, Jones MM, Lucas MA, Kanda Y, Huard J: Long-term self-renewal of postnatal muscle-derived stem cells. Mol Biol Cell 2005, 16:3323-3333.

20. Kuroda R, Usas A, Kubo S, Corsi K, Peng H, Rose T, Cummins J, Fu FH, Huard J: Cartilage repair using bone morphogenetic protein 4 and muscle-derived stem cells. Arthritis Rheum 2006, 54:433-442.

21. Zheng B, Cao B, Li G, Huard J: Mouse adipose-derived stem cells undergo multilineage differentiation in vitro but primarily osteogenic and chondrogenic differentiation in vivo. Tissue Eng 2006, 12:1891-1901.

22. Deasy BM, Lu A, Tebbets JC, Feduska JM, Schugar RC, Pollett JB, Sun B, Urish KL, Gharaibeh BM, Cao B, Rubin RT, Huard J: A role for cell sex in stem cell-mediated skeletal muscle regeneration: female cells have higher muscle regeneration efficiency. J Cell Biol 2007, 177:73-86.

23. Chirieleison SM, Feduska JM, Schugar RC, Askew Y, Deasy BM: Human muscle-derived cell populations isolated by differential adhesion rates: phenotype and contribution to skeletal muscle regeneration in Mdx/ SCID mice. Tissue Eng Part A 2012, 18:232-241.

24. Rando TA: Stem cells, ageing and the quest for immortality. Nature 2006, 441:1080-1086.

25. Sherley $J$, Stadler PB, Stadler JS: A quantitative method for the analysis of mammalian cell proliferation in culture in terms of dividing and nondividing cells. Cell Prolif 1995, 28:137-144.

26. Deasy BM, Jankowski RJ, Payne TR, Cao B, Goff JP, Greenberger JS, Huard J: Modeling stem cell population growth: incorporating terms for proliferative heterogeneity. Stem Cells 2003, 21:536-545.

27. Jankowski RJ, Deasy BM, Cao B, Gates C, Huard J: The role of CD34 expression and cellular fusion in the regeneration capacity of myogenic progenitor cells. J Cell Sci 2002, 115:4361-4374.

28. Lavasani M, Lu A, Peng H, Cummins J, Huard J: Nerve growth factor improves the muscle regeneration capacity of muscle stem cells in dystrophic muscle. Hum Gene Ther 2006, 17:180-192.

29. Espada J, Varela I, Flores I, Ugalde AP, Cadinanos J, Pendas AM, Stewart CL, Tryggvason K, Blasco MA, Freije JM, Lopez-Otin C: Nuclear envelope defects cause stem cell dysfunction in premature-aging mice. J Cell Biol 2008, 181:27-35.

30. Lavasani M, Robinson AR, Lu A, Song M, Feduska JM, Ahani B, Tilstra JS, Feldman CH, Robbins PD, Niedernhofer LJ, Huard J: Muscle-derived stem/ progenitor cell dysfunction limits healthspan and lifespan in a murine progeria model. Nat Commun 2012, 3:608.

31. Brennan TJ, Olson EN: Myogenin resides in the nucleus and acquires high affinity for a conserved enhancer element on heterodimerization. Genes Dev 1990, 4:582-595.

32. Paulin D, Li Z: Desmin: a major intermediate filament protein essential for the structural integrity and function of muscle. Exp Cell Res 2004, 301:1-7.

33. Hoffman EP, Brown RH Jr, Kunkel LM: Dystrophin: the protein product of the Duchenne muscular dystrophy locus. Cell 1987, 51:919-928.

34. Varga R, Eriksson M, Erdos MR, Olive M, Harten I, Kolodgie F, Capell BC, Cheng J, Faddah D, Perkins S, Avallone H, San H, Qu X, Ganesh S, Gordon LB, Virmani R, Wight TN, Nabel EG, Collins FS: Progressive vascular smooth muscle cell defects in a mouse model of Hutchinson-Gilford progeria syndrome. Proc Natl Acad Sci USA 2006, 103:3250-3255.

35. Zhang J, Lian Q, Zhu G, Zhou F, Sui L, Tan C, Mutalif RA, Navasankari R, Zhang Y, Tse HF, Stewart CL, Colman A: A human iPSC model of Hutchinson-Gilford progeria reveals vascular smooth muscle and mesenchymal stem cell defects. Cell Stem Cell 2011, 8:31-45.

36. Scaffidi P, Misteli T: Lamin A-dependent misregulation of adult stem cells associated with accelerated ageing. Nat Cell Biol 2008, 10:452-459.

37. Conboy IM, Conboy MJ, Wagers AJ, Girma ER, Weissman IL, Rando TA: Rejuvenation of aged progenitor cells by exposure to a young systemic environment. Nature 2005, 433:760-764.

\section{doi:10.1186/scrt183}

Cite this article as: Song et al:: Muscle-derived stem/progenitor cell dysfunction in Zmpste24-deficient progeroid mice limits muscle regeneration. Stem Cell Research \& Therapy 2013 4:33.

\section{Submit your next manuscript to BioMed Central and take full advantage of:}

- Convenient online submission

- Thorough peer review

- No space constraints or color figure charges

- Immediate publication on acceptance

- Inclusion in PubMed, CAS, Scopus and Google Scholar

- Research which is freely available for redistribution

Submit your manuscript at www.biomedcentral.com/submit
C Biomed Central 